\title{
UN METODO SPETTROSCOPICO PER LA QUANTIFICAZIONE DI FARMACI IN FLUIDI BIOLOGICI
}

\author{
PAOLO MARIA OSSI (*), et al. ${ }^{\#}$ \\ Nota presentata dal m.e. Carlo Bottani \\ (Adunanza del 6 aprile 2017)
}

\begin{abstract}
SunTO. - Viene discussa una tecnica spettroscopica innovativa per determinare la concentrazione di farmaci in fluidi biologici. Introduciamo il contesto dei farmaci caratterizzati da un ristretto indice terapeutico in relazione all'epilessia e al morbo di Parkinson. Ricapitoliamo quindi gli elementi essenziali della spettroscopia Raman e dell'intensificazione del segnale per effetto dell'interazione di un analita con una superficie metallica corrugata alla scala nanometrica. L'ottimizzazione dell'intensificazione dello spettro di un dato analita dipende dalla scelta del metallo e dai dettagli nanostrutturali della sua superficie. Consideriamo in dettaglio la sintesi di superfici di metalli nobili condotta mediante ablazione laser pulsata in gas inerte ad alta pressione. Mostriamo quindi il comportamento spettroscopico di queste superfici per determinare la concentrazione di farmaci in diversi fluidi, incluso il sangue umano. Consideriamo in particolare la carbamazepina (un farmaco antiepilettico ampiamente adottato nei Paesi in via di sviluppo) e l'apomorfina (un farmaco usato per il trattamento di pazienti affetti dal morbo di Parkinson).
\end{abstract}

$$
* * *
$$

ABSTRACT. - An innovative spectroscopic technique to determine the drug concentration in biological fluids is discussed. We introduce the context of drugs with narrow therapeutic index in relation to epilepsy and Parkinson's disease. We then recapitulate the essen-

(*) Politecnico di Milano, Dipartimento di Energia, Milano, Italy.

E-mail: paolo.ossi@polimi.it

(\#) La nota è il risultato di un lavoro di ricerca condotto in collaborazione con: Chiara Zanchi, Matteo Pistaffa, Andrea Lucotti, Matteo Tommasini, Sebastiano Trusso, Ugo De Grazia, Emilio Ciusani, Marina Casazza, Silvana Franceschetti. 
tials of Raman and enhanced Raman spectroscopy that makes use of a corrugated metallic surface. Optimizing the intensification of the spectroscopic signatures of a given analyte critically depends on the metal choice and on the fine details of the induced surface nanostructuring. We review the topic with emphasis on noble metal surfaces synthesized by pulsed laser ablation in inert gas at high pressure. The performance of optimized surfaces to determine the drug concentration in different fluids, including human blood, is discussed with reference to carbamazepine, an anti-epileptic drug widely adopted in Developing Countries and to apomorphine, a drug used to treat via subcutaneous injection patients with important manifestations of Parkinson's disease.

\section{INTRODUZIONE}

Il dosaggio di farmaci (Therapeutic Drug Monitoring, TDM) è una pratica clinica che consiste nella determinazione della concentrazione di un farmaco in un fluido biologico, solitamente il plasma sanguigno. Tale procedura è particolarmente rilevante per farmaci caratterizzati da un ristretto indice terapeutico (Narrow Therapeutic Index, NTI). In questi farmaci è minima la differenza tra la concentrazione alla quale si hanno effetti terapeutici e la concentrazione (di poco maggiore) alla quale cominciano ad essere osservati effetti collaterali negativi per il paziente (Fig. 1). È ovvio come, nelle terapie che utilizzano farmaci di questo tipo, sia rilevante per il medico poter disporre del dato di concentrazione plasmatica del farmaco. In questo modo il medico può associare le condizioni cliniche del paziente al dosaggio del farmaco in modo da garantire l'efficacia del trattamento e l'ottimizzazione della dose necessaria, così da evitare di trovarsi in condizioni per le quali si verificano effetti collaterali negativi per il paziente (Fig. 1).

L'indice terapeutico di un farmaco è rappresentato dalla distanza tra la dose necessaria per avere l'effetto farmacologico e la dose che provoca un effetto tossico. Tanto più sono distanti queste due dosi, tanto più sicuro è il farmaco, e viceversa.

TDM è utilizzato da tempo nella pratica clinica, per l'insieme ristretto dei farmaci NTI. Tra questi farmaci troviamo farmaci antitumorali ${ }^{1}$ e farmaci antiepilettici (AED, Anti Epileptic Drug), per i quali

1 S. Fornasaro, S. Dalla Marta, M. Rabusin, A. Bonifacio, V. Sergo, Toward SERS-based point-of-care approaches for therapeutic drug monitoring: the case of methotrexate, Faraday Discussions 187 (2016), 485. 
una piccola variazione della dose può essere tossica, o inefficace nel controllo dell'insorgenza delle crisi epilettiche. ${ }^{2}$

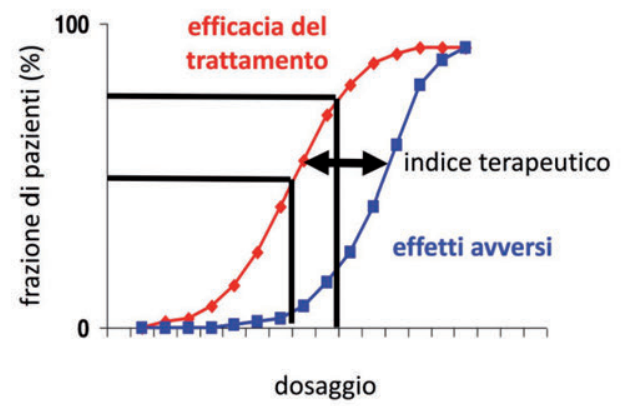

Fig. 1 - Definizione dell'indice terapeutico per un farmaco antiepilettico. Per una determinata frazione percentuale della popolazione di pazienti, l'indice terapeutico definisce l'intervallo di posologia del farmaco per il quale si osserva l'efficacia del trattamento. Tale intervallo è compreso tra la regione a bassa dose, in cui non si hanno effetti clinici, e la regione a dosi maggiori, in cui si osservano effetti clinici avversi e indesiderati. In linea di principio, più è ampia la regione compresa tra le due sigmoidi rossa e blu, più è agevole l'impiego del farmaco, dato che il dosaggio è meno critico nel determinare gli effetti clinici desiderati.

\section{EPILESSIA}

L'epilessia, tra le patologie neurologiche gravi, è la più diffusa. Il 7.6 per 1000 della popolazione del mondo presenta nel corso della sua vita una epilessia., ${ }^{3,4}$ Nella maggior parte dei casi l'epilessia può essere

2 B.W. Abou-Khalil, Making Sense of Lamotrigine Serum Levels, Epilepsy Currents 5 (2005), 115.

3 K.M. Fiest, K.M. Sauro, S. Wiebe, S.B. Patten, C.S. Kwon, J. Dykeman, T. Pringsheim, D.L. Lorenzetti, N. Jetté, Prevalence and incidence of epilepsy: A systematic review and meta-analysis of international studies, Neurology 88 (2017), 296.

4 D.J. Thurman, E. Beghi, C.E. Begley, A.T. Berg, J.R. Buchhalter, D. Ding, D.C. Hesdorffer, W.A. Hauser, L. Kazis, R. Kobau, B. Kroner, D. Labiner, K. Liow, G. Logroscino, M.T. Medina, C.R. Newton, K. Parko, A. Paschal, P.M. Preux, J.W. Sander, A. Selassie, W. Theodore, T. Tomson, S. Wiebe, and ILAE Commission on Epidemiology, Standards for epidemiologic studies and surveillance of epilepsy, Epilepsia 52 (2011), 2. 
trattata con successo, ma il gap terapeutico, cioè la differenza tra i pazienti affetti da epilessia e quelli trattati positivamente, può essere molto ampio, specialmente nei Paesi in via di sviluppo 5 anche perché parecchi farmaci sono inaccessibili, o troppo costosi. ${ }^{6}$

Inoltre, molti pazienti sottoposti a trattamento farmacologico possono soffrire di effetti collaterali che influenzano negativamente la qualità della vita, quali iperattività, stato confusionale, insonnia e depressione. ${ }^{7}$ Per ridurre l'insorgenza di effetti collaterali, ogni paziente dovrebbe assumere una dose personalizzata del farmaco, definita a partire dai risultati del dosaggio (TDM) e dall'esame critico dei dati da parte del neurologo. ${ }^{8}$

Nel caso degli AED la TDM è necessaria nelle fasi iniziali del trattamento, o a seguito di cambiamento del, o di uno dei farmaci, perché le crisi epilettiche si verificano a intervalli irregolari, per cui è difficile trovare la dose ottimale sulla sola base delle osservazioni cliniche. Inoltre, in assenza del dato di concentrazione plasmatica, i sintomi degli effetti tossici possono essere equivoci, specialmente in pazienti che soffrono di altre patologie neurologiche oltre all'epilessia.' Un altro dato rilevante che merita di essere evidenziato è che l'indice terapeutico, ovvero l'intervallo di dose adatto al trattamento di un paziente, dipende dal paziente stesso, come si osserva anche in Fig. 1. Dato un farmaco antiepilettico, non esiste una concentrazione plasmatica ottimale applicabile a ogni paziente. Alcuni pazienti possono rispondere positivamente a dosi che per altri pazienti sono insufficienti a con-

5 R. Quintas, A. Raggi, A.M. Giovannetti, M. Pagani, C. Sabariego, A. Cieza, M. Leonardi, Psychosocial difficulties in people with epilepsy: A systematic review of literature from 2005 until 2010, Epilepsy \& Behavior 25 (2012), 60.

6 A.C. Meyer, T. Dua, J. Ma, S. Saxena, G. Birbeck, Global disparities in the epilepsy treatment gap: A systematic review, Bulletin of the World Health Organization 88 (2010), 260.

7 E. Beghi, Efficacy and tolerability of the new antiepileptic drugs: comparison of two recent guidelines, The Lancet Neurology 3 (2004), 618.

8 L. Mercolini, R. Mandrioli, M. Amore, M.A. Raggi, Simultaneous HPLC-F analysis of three recent antiepileptic drugs in buman plasma, Journal of Pharmaceutical and Biomedical Analysis 53 (2010), 62.

9 S.I. Johannessen, D. Battino, D.J. Berry, M. Bialer, G. Krämer, T. Tomson, P.N. Patsalos, Therapeutic drug monitoring of the newer antiepileptic drugs, Therapeutic drug monitoring 25 (2003), 347. 
trollare l'insorgenza di crisi epilettiche. Questa situazione generale dipende dalla severità delle crisi epilettiche del paziente e dalla sua risposta farmacocinetica.

Le tecniche analitiche standard attualmente impiegate nei laboratori di analisi clinica per il dosaggio dei farmaci sono il dosaggio immunometrico (immunoassay), o varie tecniche cromatografiche come l'HPLC (High Performance Liquid Chromatography, o la cromatografia associata alla spettrometria di massa (HPLC-MS). Si tratta di tecniche specifiche e sensibili, purtroppo associate a costi elevati, tempi di attesa lunghi e necessità di prelievi di volumi sanguigni dell'ordine di alcuni millilitri. In Tab. 1 sono riportati per alcuni AED la tecnica usata per effettuare il dosaggio sanguigno, il tempo tecnico necessario per effettuarlo, il tempo richiesto per avere il risultato dal momento della consegna del campione al Laboratorio Analisi ed il costo del singolo esame. Osserviamo che la differenza tra tempo tecnico e tempo richiesto dipende dalla necessità di raccogliere (da diversi pazienti) un numero sufficiente di campioni da analizzare in gruppo per mantenere i costi entro i limiti indicati.

Tab. 1. Metodi di analisi, tempi richiesti e costi per diversi farmaci antiepilettici.

Principio attivo Metodo di analisi Tempo tecnico Tempo richiesto Costo unitario

\begin{tabular}{lcccc} 
& & (giorni) & (giorni) & (Euro) \\
\hline Oxcarbazepina & $2 ; 3$ & 2 & $3-4$ & 29 \\
\hline Perampanel & $2 ; 3$ & $3-4$ & 5 & 29 \\
\hline Lacosamide & $1 ; 2 ; 3$ & $3-4$ & 7 & 29 \\
\hline Lamotrigina & $1 ; 2 ; 3$ & $3-4$ & 7 & 29 \\
\hline Acido Valproico & $1 ; 2$ & 1 & 1 & 15 \\
\hline Levetiracetam & $1 ; 2 ; 3$ & $3-4$ & 7 & 29 \\
\hline Fenitoina & $1 ; 2 ; 3$ & 1 & 1 & 15
\end{tabular}

Metodi di analisi: 1, Immunometria; 2, spettrometria di massa tandem da fase liquida; 3, HPLC-MS Fonte: Laboratorio di Patologia Clinica e Genetica Medica, Fondazione IRCCS Istituto Neurologico C. Besta, Milano.

Queste caratteristiche intrinseche limitano la diffusione del dosaggio farmacologico e la popolazione dei pazienti che può beneficiare degli esiti di questi esami clinici. 


\section{MALATTIA Di PARKINSON}

La malattia di Parkinson (MP) è una patologia neurodegenerativa ad evoluzione lenta, ma progressiva. Essa coinvolge principalmente il controllo dei movimenti e dell'equilibrio. Tale malattia è la più frequente tra le patologie denominate "Disordini del Movimento". I sintomi di MP sono probabilmente noti da migliaia di anni (ne sono state trovate tracce in uno scritto indiano (5000 A.C.) e in uno cinese di 2500 anni fa). Il nome della malattia è legato a James Parkinson, un farmacista e chirurgo londinese che descrisse gran parte dei sintomi nel "Trattato sulla paralisi agitante" (XIX secolo). La malattia è diffusa in tutto il mondo ed in tutti i gruppi etnici. L'età media di esordio è intorno ai 5860 anni, ma circa il $5 \%$ dei pazienti può presentare un esordio giovanile tra i 21 ed i 40 anni. Prima dei 20 anni è estremamente rara. Sopra i 60 anni colpisce l'1-2\% della popolazione, mentre la percentuale sale al 3-5\% quando l'età è superiore agli $85 . .^{10,11}$

Uno dei problemi maggiori nel trattamento dei pazienti Parkinsoniani è lo sviluppo, a lungo termine, di gravi complicazioni motorie che non possono essere trattate adeguatamente con la somministrazione di farmaci per via orale a dosi tollerabili. In questi casi la somministrazione sottocutanea continua di apomorfina (APO) è un trattamento che permette di migliorare le capacità motorie dei pazienti, riducendo i periodi denominati off. ${ }^{11}$ In questo scenario clinico è fondamentale l'aggiustamento del dosaggio di APO nel paziente, tenendo conto delle variazioni di concentrazione del farmaco nel sangue (farmacocinetica). A tale scopo, nella pratica si considerano solitamente una serie di osservazioni cliniche standardizzate, unitamente, quando sia possibile effettuarli, all'esito di test sulla condizione motoria. ${ }^{12}$ In alternativa, analisi chimiche standard (HPLC-MS) su campioni sanguigni

10 Linea guida 24, Ministero della Sanità, Agosto 2013.

11 Sito italiano sulla malattia di Parkinson, Fondazione Grigioni per il Morbo di Parkinson, link consultato il 23 maggio 2017; http://www.parkinson.it/morbo-diparkinson.html

12 A.E. Elia, C. Dollenz, P. Soliveri, A. Albanese, Motor features and response to oral levodopa in patients with Parkinson's disease under continuous dopaminergic infusion or deep brain stimulation, Eur. J. Neurol. 19 (2012), 76. 
del paziente sono un metodo robusto e affidabile, ma non adottato di routine, per ottenere la concentrazione plasmatica del farmaco. Con tecniche che rendano tali misure più agevoli, ci si aspetta di poter migliorare la condizione di pazienti affetti da MP allo stadio avanzato, grazie alla possibilità di fornire informazioni utili alla determinazione della posologia ottimale e personalizzata del farmaco.

In tempi recenti, nella letteratura scientifica che tratta di dosaggio di farmaci, è emersa una tecnica promettente e complementare a quelle in uso clinico, basata sulla spettroscopia vibrazionale Raman/SERS (Surface Enhanced Raman Spectroscopy) ${ }^{13-16}$ le cui caratteristiche salienti sono discusse nel paragrafo seguente. In un possibile scenario applicativo sarebbe possibile risalire alla concentrazione plasmatica di un farmaco in campioni di origine ematica sottoposti a trattamenti minimi e rapidi (es. centrifugazione, estrazione con metanolo). La misura SERS sfrutta l'interazione della molecola di farmaco con una superficie metallica nanostrutturata e l'intensità del segnale, in condizioni controllate, permette di risalire alla quantità di molecole adsorbite sul metallo, che è funzione della concentrazione del farmaco nella soluzione in esame. Ad esempio, sono stati riportati in passato risultati che mostrano una capacità analitica della tecnica SERS comparabile con la tecnica HPLC. ${ }^{13}$ Allo stato attuale della ricerca, lo sviluppo di procedure per misure SERS quantitative richiede in ogni caso una messa a punto e validazione che si basa su determinazioni della concentrazione del farmaco condotte con metodi standard di riferimento (HPLC-MS o dosaggio immunologico). Gli sforzi attuali della ricerca nel campo SERS-TDM sono volti a superare le difficoltà

13 C. McLaughlin, D. MacMillan, C. McCardle, W. Ewen Smith, Quantitative analysis of mitoxantrone by surface-enhanced resonance Raman scattering, Analytical Chemistry 74 (2002), 3160-3167.

14 I. J. Hidi, A. Mühlig, M. Jahn, F. Liebold, D. Cialla, K. Weberac, J. Popp, LOC-SERS: towards point-of-care diagnostic of methotrexate, Analytical Methods 6 (2014), 3943-3947.

15 C. Zanchi, A. Lucotti, M. Tommasini, S. Trusso, U. de Grazia, E. Ciusani, P.M. Ossi, Laser tailored nanoparticle arrays to detect molecules at dilute concentration, Applied Surface Science 396 (2017) 1866-1874.

16 A. Jaworska, S. Fornasaro, V. Sergo, A. Bonifacio, Potential of Surface Enhanced Raman Spectroscopy (SERS) in Therapeutic Drug Monitoring (TDM). A Critical Review. Biosensors (Basel) 6 (2016), 47. 
legate alla debolezza dei segnali di alcuni farmaci e/o all'influenza negativa del segnale di fondo proveniente da quantità maggiori di biomolecole presenti insieme al farmaco nel fluido da analizzare. Un'altra importante linea di ricerca riguarda lo sviluppo di sensori SERS (ovvero superfici metalliche nanostrutturate) con caratteristiche di alta sensibilità e uniformità spaziale, controllo e riproducibilità del processo di fabbricazione e basso costo. Al momento, prima che si possa introdurre la tecnica SERS nei laboratori di analisi clinica, vi sono ancora alcune difficoltà da superare. Queste sono principalmente di natura tecnologica, per quanto riguarda le caratteristiche del sensore SERS e di natura fisico-chimica per quanto riguarda il trattamento dei campioni da sottoporre all'analisi spettroscopica. Nel seguito si studia come in due casi rappresentativi di un farmaco AED e di un farmaco AP, la tecnica SERS possa costituire un valido sistema per una determinazione veloce e minimamente invasiva della concentrazione plasmatica del farmaco. I risultati riportati in questo contributo sono volti a dimostrare le linee d'azione che sono state seguite da un team di ricercatori comprendenti fisici, chimici, ingegneri, biologi e medici, per avvicinare alla realtà clinica una tecnica spettroscopica molto promettente che finora è stata principalmente utilizzata solamente in laboratori universitari o in altri centri di ricerca scientifica.

\section{SPETTROSCOPIA RAMAN}

La spettroscopia Raman deve il suo nome a Sir Chandrasekhara Venkata Raman che pubblicò l'osservazione del fenomeno fisico alla base di questa tecnica sperimentale in uno storico articolo comparso su Nature nel 1928. ${ }^{17}$ In questo lavoro Raman e Krishnan riportavano il fatto che, a seguito dell'interazione della luce con la materia, oltre alla grande maggioranza dei fotoni diffusi in modo elastico (con la stessa energia di quelli incidenti) si osserva anche una piccola frazione di fotoni la cui energia (e quindi lunghezza d'onda) è leggermente diversa rispetto a quella della radiazione eccitatrice. Già ai suoi albori, il fenomeno fu interpretato come un processo di scambio di energia tra i fotoni incidenti e gli stati quantici del campione illuminato. In questo modo, per conservare l'ener-

17 C. V. Raman \& K. S. Krishnan, A New Type of Secondary Radiation, Nature 121 (1928), 501. 
gia totale prima e dopo l'interazione luce-materia, il fotone diffuso a seguito dell'interazione deve necessariamente cambiare la sua energia (e lunghezza d'onda) rispetto al fotone incidente. L'analisi teorica del fenomeno Raman fu essenzialmente dovuta, nel corso degli anni, ai contributi di Heisemberg, Kramers, Dirac e Placzek. Successivi contributi significativi alla teoria del fenomeno Raman in condizioni di risonanza della sorgente luminosa con transizioni elettroniche furono dovuti ad Albrecht, Peticolas, Nafie e Stein. Dal punto di vista teorico, nella descrizione dell'intensità Raman, il ruolo centrale è quello del tensore di polarizzabilità elettrica e della sua relazione con i modi normali vibrazionali della molecola, o del solido. ${ }^{18}$

La descrizione del processo Raman può essere schematizzata utilizzando il diagramma a livelli riportato in Fig. 2. Nel caso di una transizione Raman Stokes il sistema (molecola, o solido), descritto come un oscillatore armonico semplice quantistico, si trova inizialmente nello stato fondamentale, interagisce anelasticamente con il fotone incidente arrivando così in uno stato finale vibrazionale eccitato. Per la conservazione dell'energia, il fotone diffuso ha energia ( $\left.h v^{\prime}\right)$ inferiore rispetto al fotone incidente $(h v)$. La differenza di energia $h v$ - hv' è pari al quanto di energia vibrazionale $\left(h v_{\text {vib }}\right)$ dell'oscillatore che si è popolato $\left(h v-h v^{\prime}=h v_{v i b}\right)$.
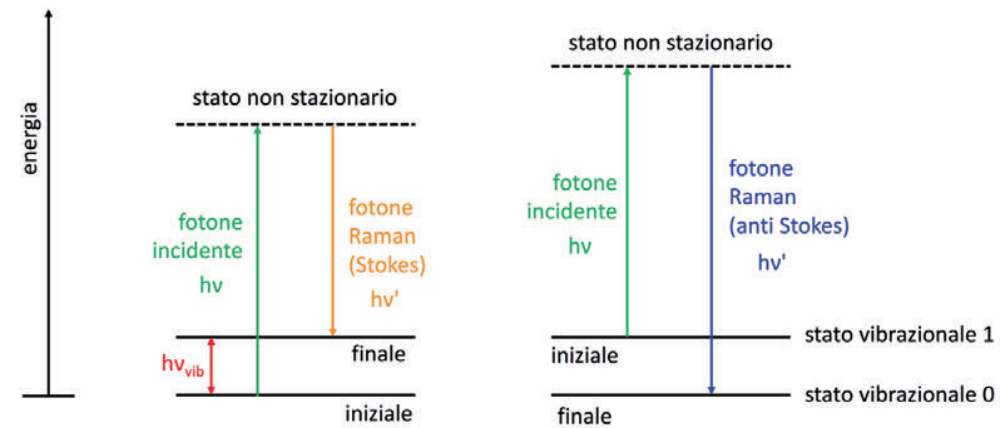

Fig. 2 - Schema dei due possibili processi di scattering Raman: Stokes (a sinistra) e antiStokes (a destra). In entrambi i casi la differenza in modulo tra l'energia del fotone incidente e quella del fotone diffuso anelasticamente è pari ad un quanto vibrazionale, $b v_{v i b \text {. }}$ Gli stati iniziale e finale dei processi Raman Stokes e anti Stokes risultano scambiati.

18 D.A. Long, The Raman Effect, Wiley, 2002. 
L'altro possibile processo Raman, definito anti-Stokes, richiede che il sistema si trovi inizialmente in uno stato vibrazionale eccitato e che nello stato finale l'energia vibrazionale del sistema sia minore di quella iniziale. In tal caso l'energia del fotone diffuso dalla molecola, o dal solido è maggiore dell'energia del fotone incidente ed il bilancio energetico risulta $h v^{\prime}-\mathrm{h} v=h v_{\mathrm{vib}}$. Dato che in condizioni ordinarie di temperatura la popolazione degli stati vibrazionali eccitati è trascurabile, nelle misure Raman si considerano solitamente le transizioni Stokes piuttosto che quelle anti-Stokes, definitamente meno intense.

Nel caso in cui l'energia del fotone incidente sia tale da poter essere assorbita dal sistema, oltre al normale processo di assorbimento di luce, si può continuare ad osservare il fenomeno Raman, che in queste condizioni prende il nome di Raman risonante e risulta intensificato rispetto alle condizioni in cui la radiazione laser non è assorbibile dal sistema. In certi casi si può arrivare ad una intensificazione del segnale di cinque ordini di grandezza rispetto alle condizioni fuori risonanza, il che corrisponde a significativi vantaggi dal punto di vista applicativo perché permette di misurare la specie risonante anche in presenza di altre specie che, di fatto, restano inosservate dato che il loro segnale è trascurabile rispetto a quello della specie risonante. Ė bene sottolineare che il processo Raman risonante non va confuso con un processo di fluorescenza, benché la fluorescenza sia un processo alternativo che può essere osservato insieme al Raman risonante e può quindi ostacolarne la misura. La differenza sostanziale tra Raman risonante e fluorescenza è che nel caso del Raman lo stato eccitato non è popolato e il processo di diffusione del fotone (scattering) è veloce $\left(10^{-14} \mathrm{~s}\right)$; nel caso della fluorescenza lo stato eccitato è popolato e successivamente decade, con un tempo di vita media del processo molto più lungo che nel caso Raman $\left(10^{-8}-10^{-5} \mathrm{~s}\right)$.

La tecnica Raman utilizza quindi la diffusione anelastica di luce monocromatica (laser) per la determinazione di transizioni vibrazionali di molecole, o solidi. In questo modo, dato il legame stretto che esiste tra gli stati vibrazionali di una molecola e la sua composizione e struttura, è possibile associare la presenza e l'intensità di specifici segnali Raman alla presenza e alla concentrazione di una data molecola nel campione analizzato. Nel caso di campioni solidi puri o quasi, la tecnica consente di riconoscere immediatamente la presenza della molecola di interesse (il principio attivo) nonostante la presenza di 
altre sostanze come gli eccipienti (si veda ad esempio il caso della molecola di Perampanel, il principio attivo del farmaco Fycompa Fig. 3). ${ }^{19}$

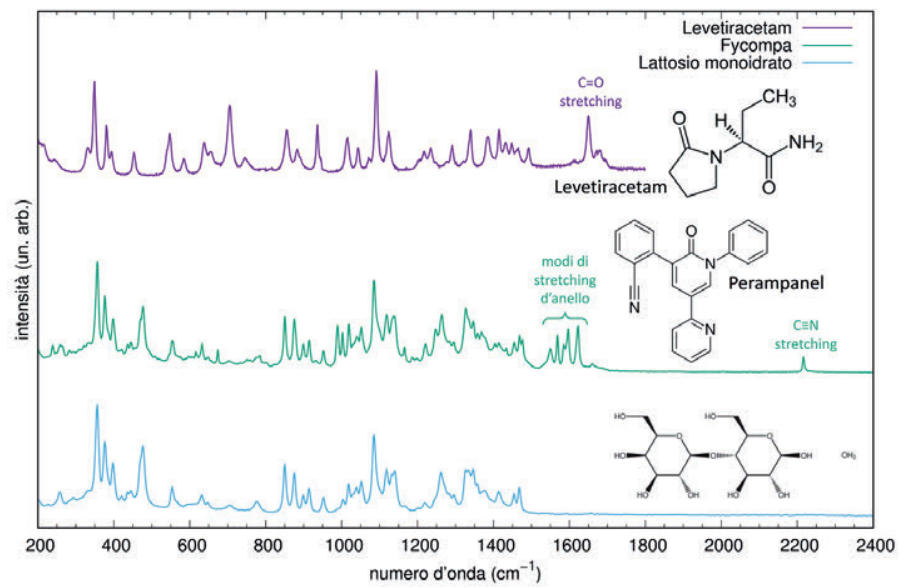

Fig. 3 - Esempi di spettri Raman di composti farmaceutici antiepilettici: (a) Levetiracetam (principio attivo del farmaco commercializzato come Keppra); (b) pastiglia del farmaco Fycompa, in cui oltre ai segnali del principio attivo Perampanel sono osservabili i segnali dell'eccipiente principale, il lattosio monoidrato (c). Sono evidenziati alcuni segnali Raman direttamente riconducibili a gruppi chimici presenti nella struttura dei due principi attivi.

Poiché le regole di selezione della spettroscopia Raman non sono le stesse che governano la spettroscopia vibrazionale di assorbimento infrarosso (IR), la tecnica Raman risulta complementare alla tecnica IR nel caratterizzare gli stati vibrazionali di molecole e solidi. Infatti, modi di vibrazione che sono deboli, o difficilmente osservabili all'IR possono risultare intensi al Raman, e viceversa.

\section{SPETTROSCOPIA RAMAN INTENSIFICATA DA SUPERFICIE - SURFACE ENHANCED RAMAN SPECTROSCOPY (SERS)}

Il limite intrinseco della spettroscopia Raman è la debolezza del

19 C. Pedrini, Development of a spectroscopic approach to antiepileptic drug sensing: a case study on lacosamide and levetiracetam, Tesi di Laurea Magistrale, Politecnico di Milano, 2017. 
segnale, ovvero la scarsità dei fotoni diffusi anelasticamente rispetto alla maggioranza dei fotoni che subiscono diffusione elastica (detta diffusione Rayleigh). Benché in condizioni di risonanza il processo Raman diventi molto più efficiente, non è sempre possibile disporre di sorgenti laser adatte a stabilire condizioni di risonanza con il sistema di interesse. Per tale motivo, fin dalla scoperta dell'effetto SERS nei primi anni '70 del 900, molti sforzi sono stati rivolti all'utilizzo dell'intensificazione prodotta da superfici corrugate di alcuni metalli per estendere significativamente il campo di applicabilità di questa tecnica spettroscopica. In particolare, lo studio di analiti presenti in soluzioni a basse concentrazioni è uno scenario particolarmente adatto alle caratteristiche della tecnica SERS. Il meccanismo di intensificazione del segnale richiede la vicinanza della molecola da analizzare ad una superficie metallica che presenti un plasmone di superficie (SP) eccitabile dall'energia dei fotoni incidenti utilizzati nell'esperimento. Un plasmone è un'eccitazione collettiva quantizzata della densità del gas di elettroni di conduzione in un solido. Un plasmone può essere eccitato colpendo con una particella carica, o con un fotone il solido. Il campo Coulombiano della prima e quello elettromagnetico (EM) del secondo provocano una ridistribuzione della carica del gas elettronico che attiva un insieme di oscillazioni di plasma, la cui energia $\left(E_{p}\right)$ dipende dalla densità di numero $(n)$ del gas elettronico

$$
E_{p}=\hbar \omega_{p}=\hbar\left(n e^{2} / \varepsilon_{0} m\right)^{1 / 2}
$$

In un metallo, per cui $n$ per la banda di conduzione è tipicamente compresa nell'intervallo $10^{27}<n<10^{29} \mathrm{~m}^{-3}, E_{p}$ è compresa fra circa $2 \mathrm{eV}$ e circa $12 \mathrm{eV}$. Se un gas elettronico semi-infinito viene confinato da una superficie piana, risolvendo l'equazione di Laplace per le fluttuazioni possibili della densità di carica si prevedono oscillazioni di plasma periodiche nel piano della superficie, la cui ampiezza decade in modo esponenziale verso l'interno del gas elettronico, normalmente alla superficie del metallo. L'unità quantizzata di tali oscillazioni è definita plasmone di superficie (surface plasmon, SP). Con le condizioni elettromagnetiche al contorno, per cui la componente tangenziale del campo EM e la componente normale dello spostamento devono essere entrambe costanti all'interfaccia fra la superficie ed il vuoto, applicando la relazione di dispersione per la costante dielettrica del 
gas elettronico, si ottiene $\mathrm{e}^{20}$ la relazione fra la frequenza di un plasmone di superficie $\omega_{\mathrm{s}}$ e quella di un plasmone di volume $\omega_{\mathrm{p}}$ :

$\omega_{\mathrm{s}}^{2}=1 / 2 \omega_{\mathrm{p}}^{2}$

Le intensità relative delle eccitazioni plasmoniche di volume e di superficie dipendono da angolo di incidenza, energia, e polarizzazione della radiazione EM eccitatrice.

Quando le dimensioni tipiche delle strutture metalliche scendono alla scala nanometrica $(5-50 \mathrm{~nm})$, a causa di fenomeni quantistici che intervengono alle piccole dimensioni, si osserva uno spostamento delle frequenze plasmoniche a più basse energie. Nanoparticelle (NP) di argento e oro presentano i picchi di assorbimento relativi alla presenza di SP (associati alle oscillazioni di plasma degli elettroni di conduzione alla superficie della particella) nel visibile, rispettivamente attorno a 410 $\mathrm{nm}$ e $520 \mathrm{~nm}$. Ciò rende possibile eccitare tali oscillazioni elettroniche utilizzando sorgenti laser operanti nello spettro del visibile. Quando un analita venga adsorbito alla superficie di nanostrutture metalliche, nel caso in cui la frequenza della radiazione EM incidente sia in risonanza con la frequenza del SP, si verifica un drammatico innalzamento dell'efficienza Raman indotto dalle oscillazioni degli elettroni di superficie. Infatti, queste oscillazioni generano una ridistribuzione del campo EM sulla superficie della NP amplificandolo di parecchi ordini di grandezza e amplificando allo stesso tempo l'efficienza Raman. Nel caso di singole NP l'innalzamento del segnale Raman è sufficiente alla rivelazione di molecole con sezione d'urto Raman molto bassa, ma l'effetto è ancora più eclatante nel caso in cui la molecola venga adsorbita nello spazio (gap) tra due NP fra loro adiacenti: qui la risonanza produce innalzamenti del segnale Raman dell'ordine di fattori $10^{8-10}$, tali da consentire la rivelazione, in casi favorevoli, di singole molecole. Ricordiamo che il fattore di intensificazione (enhancement) Raman $Q$ è dato da:

$$
Q_{\text {SERS }}=\left|\frac{E\left(\mathrm{r}, \omega_{0}\right)}{E\left(\omega_{0}\right)}\right|^{4}
$$

20 H.M. Rosenberg, The Solid State, Clarendon Press, Oxford, UK, 1974. 
dove $E\left(r, \omega_{0}\right)$ è il campo elettromagnetico totale alla posizione della molecola sulla nanostruttura e $\mathrm{E}\left(\omega_{0}\right)$ è il campo elettromagnetico associato alla radiazione incidente. Oltre a questo meccanismo elettromagnetico di intensificazione del segnale Raman, si osserva in molti casi un ulteriore e spesso benefico innalzamento del segnale causato dall'interazione chimica dell'analita con la superficie metallica. Tale interazione è usualmente accompagnata da un trasferimento di carica tra metallo e analita e dalla possibilità che l'eccitatrice utilizzata nell'esperimento possa promuovere transizioni elettroniche metallo-analita, per le quali si possono instaurare le grandi polarizzabilità di transizione che sono all'origine dell'intensificazione dell'intensità Raman. I metalli nobili nanostrutturati (specialmente $\mathrm{Ag}$ ed $\mathrm{Au}$ ) sono sistemi molto popolari nelle misure SERS perché presentano SP eccitabili con la radiazione nel visibile di alcuni dei laser più comunemente utilizzati. Essi, rispetto ad altri metalli, mostrano gli effetti di intensificazione maggiore del segnale Raman. In molti casi, per entrambi i metalli, è stato osservato anche l'effetto di intensificazione chimico.

\section{SUBSTRATI SERS REALIZZATI CON DEPOSIZIONE A LASER PULSATO}

La posizione (frequenza) e larghezza del SP sono le caratteristiche ottiche di specifico interesse di una superficie metallica corrugata quando se ne voglia esplorare il comportamento utilizzandola come substrato con attività SERS. Definita la scelta del metallo, caduta sui metalli nobili argento $(\mathrm{Ag})$ e oro $(\mathrm{Au})$ che fin dalla scoperta dell'effetto SERS sono noti per presentare i più cospicui incrementi di intensità dei segnali Raman, in linea di principio il problema consiste nell'ingegnerizzare la nanostruttura della superficie, controllandone lo sviluppo così da ottenere i massimi incrementi di segnale. La tecnica da noi utilizzata per realizzare una superficie corrugata artificialmente alla scala nanometrica è l'ablazione a laser pulsato (PLA, pulsed laser ablation) effettuata in un gas ambiente chimicamente inerte ad alta pressione. Schematicamente, un impulso laser di durata fra 1 e 10 ns viene focalizzato su un'area della superficie del bersaglio e ivi deposita una certa densità di energia. Questa provoca riscaldamento ed evaporazione di atomi e piccoli cluster dalla superficie irraggiata; il fenomeno è descritto dall'equazione di Hertz-Knudsen per l'evaporazione normale. Se la densità di energia depositata dal laser al bersaglio supera la soglia defi- 
nita di ablazione, la quantità di materia espulsa dalla superficie cresce tanto da costituire un vapore denso, in cui prevalgono ancora atomi e piccoli cluster neutri, inizialmente posizionato sopra l'area irraggiata. Tale vapore è molto anisotropo, dato che esso ha circa la stessa superficie dell'area irraggiata, il cui raggio è molto superiore allo spessore. Con bersagli metallici la formazione del vapore ablato richiede un tempo molto minore (qualche $10^{-2}$ ) della durata dell'impulso laser che lo ha prodotto. Tale nuvola di vapore assorbe fortemente la radiazione laser: il parametro significativo qui è la densità di numero delle particelle ablate $\mathrm{n}_{\mathrm{a}}$, il cui valore critico $\mathrm{n}_{\mathrm{cr}}$ è

$\mathrm{n}_{\text {cr }}=\left(4 \pi \varepsilon_{0} \mathrm{~m}_{\mathrm{e}} \mathrm{e}^{-2} \mathrm{c}^{2}\right) / \lambda^{2}$

ove $\mathrm{m}_{\mathrm{e}}$ ed e sono massa e carica dell'elettrone a riposo, $\varepsilon_{0}$ la costante dielettrica e c la velocità della luce nel vuoto, $\lambda$ la lunghezza d'onda della radiazione laser. Se $n_{a}$ è maggiore di $n_{c r}$ il vapore è opaco rispetto alla radiazione laser e la assorbe, venendone energizzato attraverso i meccanismi di ionizzazione diretta a singolo fotone e di bremsstrablung inversa degli elettroni liberi nel campo degli ioni. Contemporaneamente il bersaglio viene schermato rispetto all'azione dell'impulso laser. Il vapore energizzato viene convertito da neutro a ionizzato, con grado di ionizzazione 1 , diventando un plasma da ablazione molto energetico (la temperatura elettronica equivalente $T_{e}$ è compresa fra $2 \mathrm{eV}$ e $10 \mathrm{eV}$ ) in espansione supersonica pilotata da forti gradienti di pressione normali alla superficie del bersaglio. Tale plasma, per analogia suggerita dalla sua forma, osservata in immagini di fotografia ultra-rapida, viene chiamato piuma di ablazione. Se la propagazione della piuma avviene in un gas inerte, al crescere della pressione di quest'ultimo, si sviluppa un'onda d'urto al fronte di propagazione della piuma, evidente da fotografie della piuma stessa, la piuma viene frenata, la sua forma diviene semi-sferica, le dimensioni sono ridotte rispetto a quelle osservate in espansioni in vuoto e si instaura un comportamento di propagazione diffusiva a tempi lunghi (microsecondi) contemporaneamente al moto retrogrado della coda della piuma verso il bersaglio. Questo comportamento, tipico di espansioni in gas massivi a pressioni fra 30 e $70 \mathrm{~Pa}$, comporta anche una significativa inter-penetrazione fra specie del plasma ed atomi del gas ambiente che porta alla separazione della piuma in due gruppi di particelle: un gruppo veloce che propaga attraverso il gas con velocità simile a quella che avrebbe espandendosi 
in vuoto praticamente senza collisioni ed un gruppo lento, ritardato a causa delle interazioni collisionali subite con gli atomi del gas.

A partire da una rassegna critica ${ }^{21}$ dei modelli che sono stati proposti per descrivere la complessa fenomenologia appena sintetizzata, abbiamo elaborato un modello per l'espansione in un gas ad alta pressione di un plasma da ablazione, nel cui ambito si prevede il numero $\mathrm{N}$ di atomi costituenti una NP che si formi nella piuma durante la fase collisionale della sua espansione:

$\mathrm{N}=\left(<\mathrm{n}_{\mathrm{a}}>\sigma_{\mathrm{a}-\mathrm{a}}<\mathrm{v}>\mathrm{t}_{\mathrm{f}}\right) \times\left(<\mathrm{n}_{\mathrm{g}}>\sigma_{\mathrm{a}-\mathrm{g}}<\mathrm{v}>\mathrm{t}_{\mathrm{f}}\right)$

$n$ indica la densità di numero, $\sigma$ la sezione d'urto di collisione elastica, $<\mathrm{v}>$ la velocità media delle particelle e $\mathrm{t}_{\mathrm{f}}$ rappresenta il tempo di formazione delle NP nella piuma. I pedici a e g si riferiscono alle specie ablate e agli atomi del gas ambiente.

Una ricerca sistematica ci ha indicato i parametri di processo rilevanti per l'ablazione laser e per la successiva deposizione su un supporto rigido inerte di un numero crescente di piume di ablazione fra loro statisticamente uguali che dia luogo ad un substrato costituito da NP isolate, poi progressivamente interagenti tanto da coalescere e formare strutture sempre più estese, fino alla realizzazione di un film semi-continuo. Per definite condizioni sperimentali, quali lunghezza d'onda della radiazione laser, durata temporale ed energia degli impulsi, angolo di incidenza dell'impulso laser sul bersaglio, distanza bersaglio-supporto, natura del gas ambiente, temperatura di processo, i due parametri rilevanti per definire la nano-struttura di superficie del substrato in fase di deposizione sono il numero di impulsi laser $\mathrm{N}_{\mathrm{LP}}$ e la pressione del gas ambiente $\mathrm{p}_{\mathrm{g}}$. $\mathrm{Al}$ crescere di $\mathrm{p}_{\mathrm{g}}$, mantenendo costante $\mathrm{N}_{\mathrm{LP}}$, la superficie appare ricoperta da nanoparticelle progressivamente più piccole, con forma sempre più regolare, sferica. Il valore di $\mathrm{N}_{\mathrm{LP}}$ definisce il grado di ricopertura del substrato. Al crescere di $\mathrm{N}_{\mathrm{LP}}$, mantenendo costante $\mathrm{p}_{\mathrm{g}}$, si osserva principalmente un aumento della densità spaziale della popolazione di NP sul supporto; al progredire della coalescenza si ha una progressiva deviazione della loro forma da sferica verso geometrie più irregolari. Quanto alle proprietà ottiche delle nanostrutture ottenute, la

21 F. Neri, P.M. Ossi, S. Trusso, Cluster synthesis and assembling in laser generated plasmas, Rivista del Nuovo Cimento 34 (2011), 103. 
frequenza del SP aumenta, per fissato valore di $\mathrm{N}_{\mathrm{LP}}$, al crescere di $\mathrm{p}_{g}$, così come, a fissata $\mathrm{p}_{\mathrm{g}}$, quando diminuisce $\mathrm{N}_{\mathrm{LP}}$.

In Fig. 4 questo andamento è illustrato nel caso di arrangiamenti auto-assemblati di NP di Ag su supporti di (100)Si. Per NP di Au si osservano gli stessi andamenti parametrici appena discussi per NP di $\mathrm{Ag}$. L'osservazione con microscopia elettronica in trasmissione (TEM) di assemblee di NP dei due metalli nobili, depositate in condizioni tali da evitare coalescenza, indica intervalli di dimensioni fra 1 e $4 \mathrm{~nm}$ per Ag e fra 2 e $6 \mathrm{~nm}$ per Au. I dati sono in accordo con le previsioni modellistiche (eq. 5). Siamo quindi in grado di controllare la formazione di tali NP e la loro deposizione in condizioni di integrità sul supporto ove formano un substrato con definite proprietà ottiche.

(a)

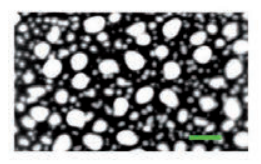

(b)

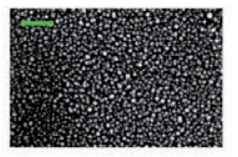

$\mathrm{p}_{\mathrm{g}}$ cresce (c)

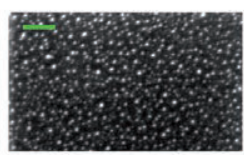

(d)
$\mathrm{N}_{\mathrm{LP}}$ cresce

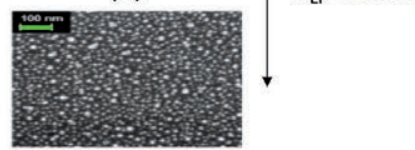

Fig. 4 - Micrografie SEM che mostrano la dipendenza della nanostruttura di superficie di un film di argento ablato in argon dalla pressione del gas ambiente $\left(p_{g}\right)$ e dal numero di impulsi laser $\left(N_{L P}\right)$. In (a), (b), (c) $N_{L P}=15000 ; p_{g}=10 \mathrm{~Pa}(a), 40 \mathrm{~Pa}(b), 70 \mathrm{~Pa}(\mathrm{c})$. In (d) $p_{g}=70 \mathrm{~Pa}, N_{L P}=30000$. Le barre verdi corrispondono a $100 \mathrm{~nm}$.

Rimane un parametro dell'ablazione parzialmente nascosto che influisce significativamente sulla nanostruttura di un film: la massa ablata da ogni impulso laser. Questa dipende, a parità di densità di energia depositata dall'impulso, dall'area su cui il fascio laser è focalizzato. Abbiamo studiato specificamente questo problema, considerando sia la propagazione della piuma, ${ }^{22}$ sia la nanostruttura di superficie e le proprietà ottiche di un film. ${ }^{23}$ Con impulsi che producono masse ablate

22 M.C. Spadaro, E. Fazio, F. Neri, S. Trusso, P.M. Ossi, On the role of the ablated mass on the propagation of a laser-generated plasma in an ambient gas, Europhys. Lett. 109 (2015), 25002.

23 M.C. Spadaro, E. Fazio, F. Neri, P.M. Ossi, S. Trusso, On the influence of the 
in rapporto circa 1:2 (7.0 ng, $\mathrm{M}_{\mathrm{p}} ; 16.4 \mathrm{ng}, \mathrm{M}_{\mathrm{g}}$ ) si ottengono film di $\mathrm{Ag}$ morfologicamente simili, con nanostrutture a isole separate fra loro da canali di larghezza ed estensione variabili. Tuttavia, i film hanno proprietà ottiche molto differenti: nel caso della massa ablata $M_{p}$ il SP presenta un evidente massimo a circa $590 \mathrm{~nm}$, mentre si osserva un largo massimo a $820 \mathrm{~nm}$ nel caso della massa ablata $\mathrm{M}_{\mathrm{g}}$. Una verifica delle prestazioni SERS dei due substrati con un analita di riferimento, Rodamina 6G (R6G) in soluzioni acquose a concentrazione molare moderata $\left(10^{-6} \mathrm{M}\right)$, usando eccitatrice di lunghezza d'onda $785 \mathrm{~nm}$, mostra che il film depositato con $\mathrm{M}_{\mathrm{g}}$ non produce segnali apprezzabili dell'analita, a differenza di quello preparato con $\mathrm{M}_{\mathrm{p}}$, con il quale si ottiene uno spettro SERS ben definito.

In conclusione, siamo in grado di sintetizzare mediante ablazione laser in gas inerte ad alta pressione substrati SERS attivi di cui modificare la nanostruttura di superficie e, di conseguenza, le proprietà ottiche, in funzione delle caratteristiche dello spettro Raman dell'analita di interesse.

\section{APPLICAZIONE DELLA SPETTROSCOPIA SERS ALLA MISURA DELLA CONCENTRAZIONE DI FARMACI}

\section{Condizioni sperimentali}

Gli esempi di seguito riportati riguardano l'applicazione della spettroscopia SERS, che fa uso di substrati nanostrutturati prodotti con la tecnica PLD sopra descritta, alla determinazione della concentrazione di farmaci in soluzione. Il dispositivo sperimentale, illustrato in Fig. 5, prevede poche e semplici operazioni che precedono la misura SERS. In particolare, il substrato nanostrutturato, utilizzato come sensore SERS, viene immerso nella soluzione di farmaco ad una data concentrazione (fase dip), lasciato in contatto con tale soluzione per un tempo fissato, asciugato (fase $d r y$ ), ed infine posto sotto l'obiettivo dello spettrometro Raman, dove viene acquisito lo spettro SERS.

mass ablated by a laser pulse on thin film morphology and optical properties, Applied Physics A 117 (2014), 137-142. 

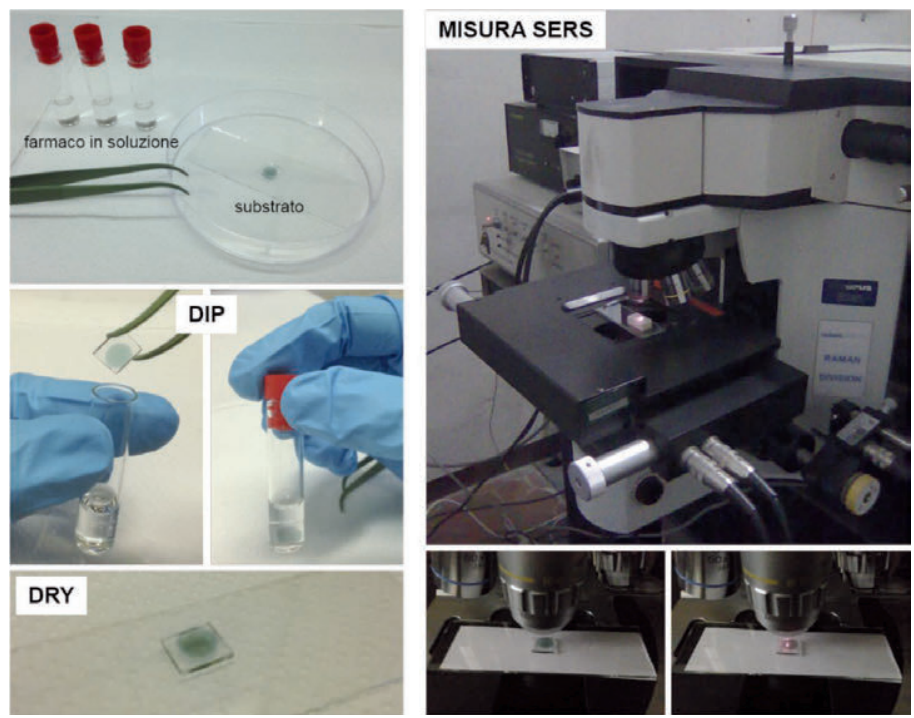

Fig. 5 - Dispositivo sperimentale. I pannelli di sinistra mostrano un substrato di oro nanostrutturato, deposto su vetro, tra quelli utilizzati per le misure SERS dei farmaci qui considerati. Il substrato viene immerso in un volume noto di soluzione di farmaco da analizzare, ad una data concentrazione, lasciato in contatto con la soluzione per un tempo fissato, quindi lasciato asciugare prima della misura SERS. I pannelli di destra mostrano la misura SERS, con uno strumento da laboratorio Jobin-Yvon HR800.

\section{Messa a punto del metodo}

Dato un insieme omogeneo di film nanostrutturati ottenuti mediante PLD, la messa a punto del metodo SERS richiede una serie di indagini chimico-fisiche, preliminari all'applicazione quantitativa, che consentono di utilizzare con sicurezza i segnali SERS di riferimento del farmaco in soluzione e di ottimizzare l'intensità di tali segnali. Tale serie di indagini richiede:

1) l'acquisizione di uno spettro Raman di riferimento per il farmaco in un dato solvente, ed il confronto con lo spettro SERS dello stesso farmaco in soluzioni diluite. Ciò permette l'assegnazione dei picchi principali nello spettro SERS, ovvero l'attribuzione a specifici modi normali di vibrazione dei principali segnali del farmaco oggetto di indagine.

2) La selezione, sulla base dello spettro SERS raccolto, di indicatori (markers) spettrali robusti, allo scopo di riconoscere e quantificare l'analita (farmaco), anche in matrici via via più complesse, dove la 
concomitante presenza di altre specie può dar luogo ad una risposta Raman/SERS (caso di biomolecole presenti insieme al farmaco nel fluido da analizzare) non trascurabile.

3) L'ottimizzazione del protocollo dip $E d r y$, per la scelta migliore dei tempi di contatto del substrato (sensore) con la soluzione contenente il farmaco ad una data concentrazione.

4) L'ottimizzazione dei parametri di acquisizione dello spettro SERS, quali il tempo di integrazione, la potenza del laser e le dimensioni dell'area irraggiata, per avere spettri con un buon rapporto segnale/rumore, anche a basse concentrazioni del farmaco, pur senza rinunciare ad una misura rapida, prerequisito fondamentale per l'applicazione di interesse.

5) La verifica della riproducibilità e dell'uniformità spaziale del segnale SERS dell'analita. L'area di misura (tipicamente dell'ordine di $1 \mu \mathrm{m}$ di diametro) è infatti molto più piccola della superficie dei sensori utilizzati (pochi $\mathrm{mm}$ di diametro), e la correlazione tra l'intensità del segnale SERS e la concentrazione del farmaco misurato è valutata sulla base di una media spaziale dell'intensità/area di picchi selezionati (a partire da un numero sufficiente di misure, esplorando la superficie del sensore).

\section{Applicazione 1: Apomorfina}

La prima applicazione riguarda lo sviluppo di un metodo per la quantificazione di apomorfina (APO) in soluzione acquosa mediante misure SERS, e la successiva prova di applicabilità del metodo alla rilevazione di APO in campioni di origine clinica. Il risultato finale è stato quello di ottenere una risposta SERS di APO ad una concentrazione limite inferiore di $3.3 \times 10^{-7} \mathrm{M}$ in soluzione acquosa, e di $3.3 \times 10^{-6} \mathrm{M}$ in siero.

APO, (6aR)-6-metil-5,6,6a,7-tetraidro-4H-dibenzo[de,g]chinolina-10,11-diolo, è un farmaco utilizzato per il trattamento del morbo di Parkinson. La sua struttura chimica è mostrata di seguito:<smiles>CN1CCc2cccc3c2C1Cc1ccc(O)c(O)c1-3</smiles> 
Nel caso di pazienti con gravi complicazioni (in una fase avanzata della malattia) l'infusione continua sottocutanea rappresenta una valida alternativa alla somministrazione orale. Il dosaggio di APO per via spettroscopica (SERS), da campione di sangue, o plasma, rappresenta una sostanziale innovazione nella pratica clinica, poiché attualmente la ricerca dei livelli del farmaco avviene a partire dall'osservazione clinica del paziente (test di condizione motoria).

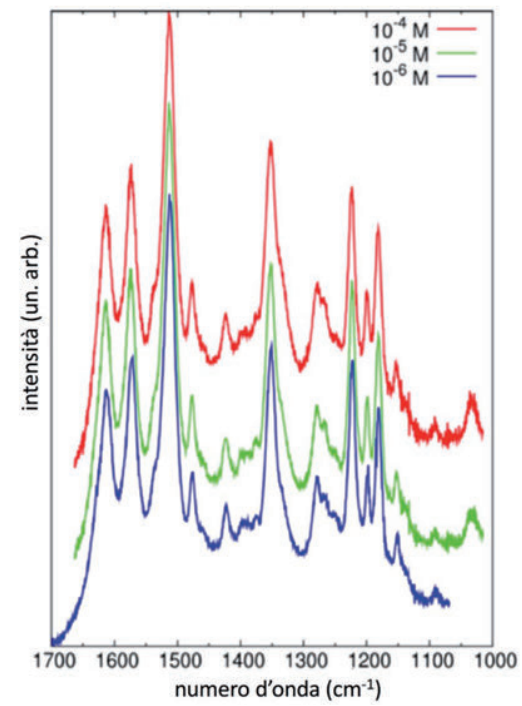

Fig. 6 - Spettri SERS di Apomorfina in soluzione acquosa, ottenuti da substrato di oro nanostrutturato a tre diverse concentrazioni molari $\left(10^{-4}, 10^{-5}\right.$ e $\left.10^{-6} \mathrm{M}\right)$. Gli spettri sono stati acquisiti con uno strumento da laboratorio Jobin-Yvon HR800, utilizzando un laser a stato solido a $785 \mathrm{~nm}$, e un obiettivo 50x. Il tempo di integrazione è di $600 \mathrm{~s}$.

La Fig. 6 mostra una serie di spettri SERS di APO in soluzione acquosa, a tre diverse concentrazioni molari $\left(10^{-4}, 10^{-5}\right.$ e $\left.10^{-6} \mathrm{M}\right){ }^{24} \mathrm{Il}$ segnale SERS è ottenuto utilizzando substrati nanostrutturati di oro prodotti con $\mathrm{N}_{\mathrm{LP}}=3 \times 10^{4}$ in atmosfera di Ar, alla pressione parziale di $100 \mathrm{~Pa}$. L'esperimento, condotto in condizioni tali da massimizzare il

24 N. Agarwal, E. Fazio, F. Neri, S. Trusso, C. Castiglioni, A. Lucotti, N. Santo, P.M. Ossi, Ag and Au nanoparticles for SERS Substrates Produced by Pulsed Laser Ablation, Crystal Research and Technology 46 (2011), 836-840. 
segnale dell'analita (durata della fase dip pari a 60 minuti), dimostra l'elevata sensibilità dei substrati considerati nei confronti del farmaco, anche alle basse concentrazioni. Il segnale SERS di APO è riproducibile alle tre diverse concentrazioni, e in particolare è possibile raggiungere la concentrazione di interesse $\left(10^{-6} \mathrm{M}\right)$.

In una fase successiva è stato possibile verificare la capacità del metodo di discriminare, attraverso la valutazione di differenze nelle intensità degli spettri SERS, la concentrazione di farmaco in soluzione. Sono stati utilizzati, con risultati promettenti, substrati SERS attivi di oro prodotti con $\mathrm{N}_{\mathrm{LP}}=1 \times 10^{4}$ in atmosfera di Ar, alla pressione parziale di $100 \mathrm{~Pa}$, per la rilevazione di APO in soluzione acquosa in un intervallo di concentrazione compreso tra $3.3 \times 10^{-4}$ e $3.3 \times 10^{-7} \mathrm{M}$. I substrati sono stati immersi in un volume noto di soluzione per un tempo considerevolmente ridotto rispetto alla fase precedente. In particolare, per la fase dip è stata selezionata una durata ottimale di 5 minuti.

I picchi, selezionati tra i più intensi caratteristici dello spettro SERS di APO, utilizzati per correlare l'intensità alla concentrazione, sono il picco a $1353 \mathrm{~cm}^{-1}$, e il picco a $1515 \mathrm{~cm}^{-1}$.

Mentre alla più bassa delle concentrazioni considerate $\left(3.3 \times 10^{-7} \mathrm{M}\right)$ si osserva solo un debole segnale dell'analita a $1515 \mathrm{~cm}^{-1}$, e tempi di integrazione più alti non permettono di migliorare il rapporto segnale/rumore dello spettro, a partire dalla concentrazione di $3.3 \times 10^{-6} \mathrm{M}$ lo spettro di $\mathrm{APO}$ è di buona qualità, e l'intensità dei picchi aumenta all'aumentare della concentrazione del farmaco. L'andamento della risposta SERS del sensore di oro alle diverse concentrazioni di farmaco è illustrata, attraverso il confronto di spettri rappresentativi, in Fig. $7 .^{25}$ Dall'analisi di tali spettri è possibile ricavare un andamento, caratteristico, della risposta del sensore (intensità SERS) in funzione della concentrazione di APO.

L'ottimizzazione del numero di impulsi nella produzione mediante PLD dei substrati di oro, ha permesso infine di produrre sensori con caratteristiche superiori, in grado di mostrare una risposta SERS intensa per APO in soluzione acquosa alla concentrazione di $3.3 \times 10^{-7} \mathrm{M}$. In Fig. 8 è mostrata la morfologia rappresentativa di questa classe di substrati SERS attivi ottenuti mediante PLD.

25 C. Zanchi, A. Lucotti, M. Tommasini, S. Trusso, U. de Grazia, E. Ciusani, P.M. Ossi, Au nanoparticle-based sensor for apomorphine detection in plasma, Beilstein Journal of Nanotechnology 6 (2015), 2224-2232. 


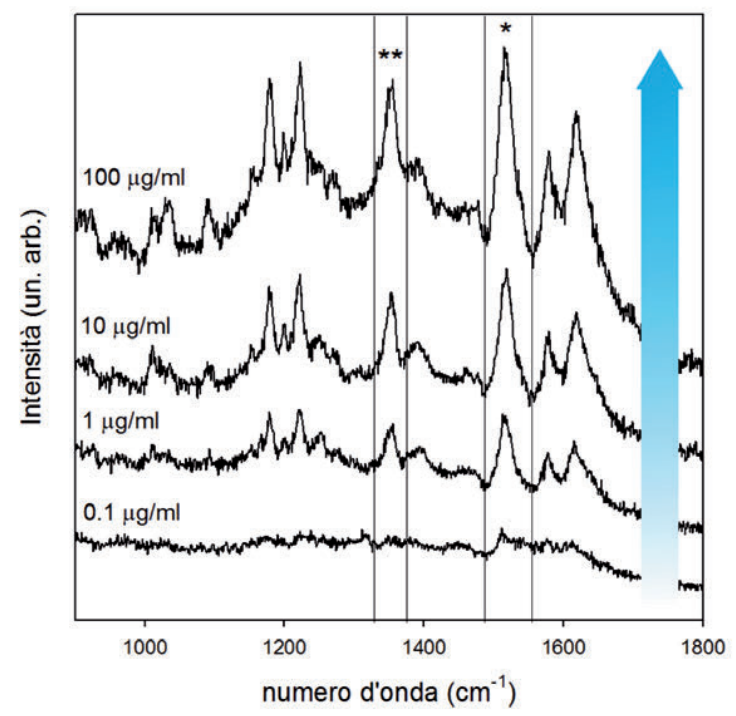

Fig. 7 - Spettri SERS di soluzioni acquose di Apomorfina a 100, 10, 1, $0.1 \mu \mathrm{g} / \mathrm{mL}$ (corrispondenti a concentrazioni da $3.3 \times 10^{-4} \mathrm{M}$ a $3.3 \times 10^{-7} \mathrm{M}$ ) ottenuti con un tempo di immersione di 5 minuti. I picchi contrassegnati con gli asterischi a $1515 \mathrm{~cm}^{-1}$ e $1353 \mathrm{~cm}^{-1}$ sono $i$ marker spettrali selezionati ai fini dello studio della dipendenza del segnale dalla concentrazione di analita.

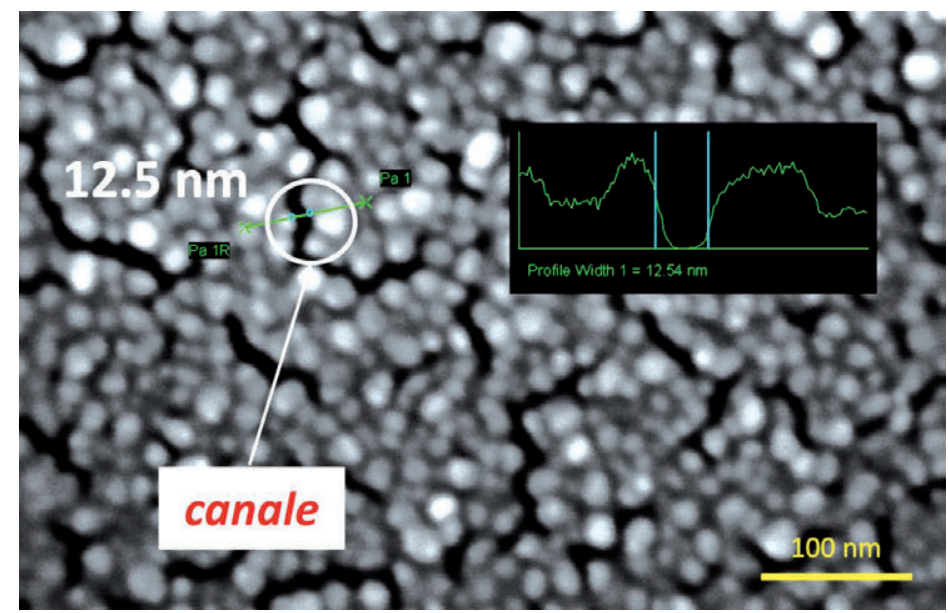

Fig. 8-Micrografia SEM rappresentativa di un substrato di nanoparticelle di oro ottenuto con $10^{4}$ impulsi laser, in Ar alla pressione parziale di $100 \mathrm{~Pa}$. 
La Fig. 9 mostra il confronto fra gli spettri SERS di APO alla concentrazione di $6.6 \times 10^{-5} \mathrm{M}$ in plasma sanguigno e $3.3 \times 10^{-6} \mathrm{M}$ in siero. Gli spettri sono stati ottenuti dalle due nanostrutture di oro con $\mathrm{N}_{\mathrm{LP}}$ rispettivamente pari a $1 \times 10^{4}$ e $2 \times 10^{4} \cdot{ }^{26} \mathrm{Nel}$ campione di siero sono riconoscibili tutte le caratteristiche spettrali principali dello spettro SERS di APO. In modo analogo a quanto già osservato in soluzione acquosa, il substrato deposto con un numero maggiore di impulsi laser mostra segnali meglio definiti e più intensi, benché quello preparato con $\mathrm{N}_{\text {LP }}$ minore sia stato utilizzato per analizzare una concentrazione di APO più alta di oltre un ordine di grandezza. La minore sensibilità del metodo nei confronti di APO in campione biologico, rispetto ai risultati in soluzione acquosa, è data dalla presenza, nel siero, di proteine (in particolare albumina), che legano una frazione importante di APO (fino al $70 \%$ ), rendendone di fatto la concentrazione libera inferiore di quasi un ordine di grandezza rispetto a quella nominale.

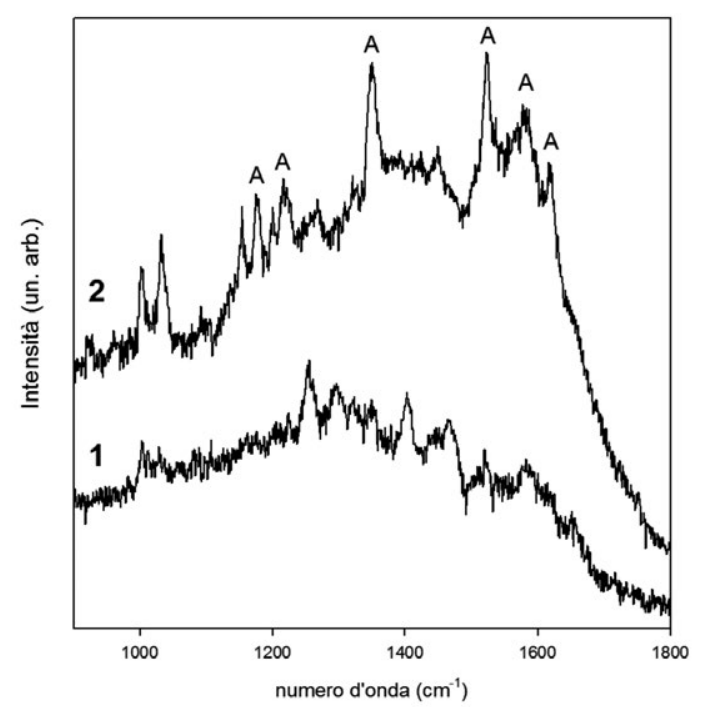

Fig. 9 - Confronto tra lo spettro SERS di apomorfina in plasma $\left(6.6 \times 10^{-5} \mathrm{M}\right.$; curva 1) acquisito da substrato di oro deposto con numero di impulsi laser pari a $1 \times 10^{4}$ (pressione parziale di $\mathrm{Ar}, 100 \mathrm{~Pa}$ ), e lo spettro SERS di apomorfina in siero (3.3 $10^{-6} \mathrm{M}$; curva 2) acquisito da substrato di oro ottimizzato rispetto al numero di impulsi laser $\left(2 \times 10^{4}\right.$, pressione parziale di Ar, $100 \mathrm{~Pa}$ ).

26 S. Trusso, C. Zanchi, A. Bombelli, A. Lucotti, M. Tommasini, U. de Grazia, 


\section{Applicazione 2: Carbamazepina}

Il secondo esempio riguarda lo studio della risposta SERS di Carbamazepina (CBZ) adsorbita su substrato di oro nanostrutturato, e la realizzazione di una curva di risposta del sensore (curva di calibrazione), in funzione della concentrazione di CBZ in metanolo. La CBZ, benzo[b][1] benzazepina-11-carbossamide, è un farmaco anti-epilettico largamente impiegato nei Paesi in via di sviluppo. La struttura chimica di CBZ è la seguente:

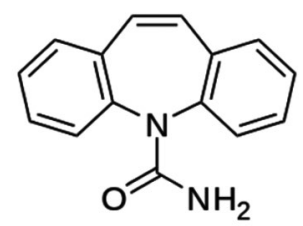

L'ottimizzazione delle condizioni di deposizione nella produzione mediante PLD di substrati nanostrutturati di oro su supporti di vetro (vedi Applicazione 1) ha permesso di ottenere un buon segnale SERS del farmaco anti-epilettico, e di attribuirne i segnali principali. In particolare, la Fig. 10 riporta il confronto tra lo spettro SERS di CBZ ed il suo spettro Raman in metanolo $(\mathrm{MeOH})$. I segnali SERS più intensi di CBZ si trovano nella finestra spettrale $650-1700 \mathrm{~cm}^{-1}$. I modi SERS attivi principali, ovvero i picchi a 1619, 1599, 1564, 1309, 1222, 718, $697 \mathrm{~cm}^{-1}$ trovano una corrispondenza con lo spettro Raman di CBZ in $\mathrm{MeOH}$, e possono essere tutti assegnati a vibrazioni caratteristiche della molecola di farmaco sulla base di calcoli DFT ${ }^{15}$. Differenze di pochi numeri d'onda dei segnali SERS rispetto a quelli Raman vengono attribuite al differente intorno chimico sperimentato da CBZ su oro rispetto a CBZ in soluzione. Tali piccole differenze confermano anche la debole interazione tra la CBZ e la superficie di oro SERS-attiva.

Proprio l'interazione debole tra il farmaco e la superficie di oro del sensore, ha reso possibile lo sviluppo di un metodo basato sull'utilizzo di un unico sensore, lavabile, per ottenere una curva di calibra-

E. Ciusani, L.M. Romito, P.M. Ossi, Synthesis by pulsed laser ablation of 2D nanostructures for advanced biomedical sensing, Journal of Instrumentation 11 (2016), C05006. 
zione di CBZ in $\mathrm{MeOH}$ nella finestra di concentrazioni di interesse clinico $(6-12 \mathrm{mg} / \mathrm{l})$. Si è dimostrato infatti che, dopo opportuno lavaggio con $\mathrm{MeOH}$, il sensore è riutilizzabile, ed è stata verificata la proporzionalità (linearità) tra la risposta SERS di CBZ e la sua concentrazione in $\mathrm{MeOH}$, nell'intervallo di concentrazioni 6-50 mg/l.
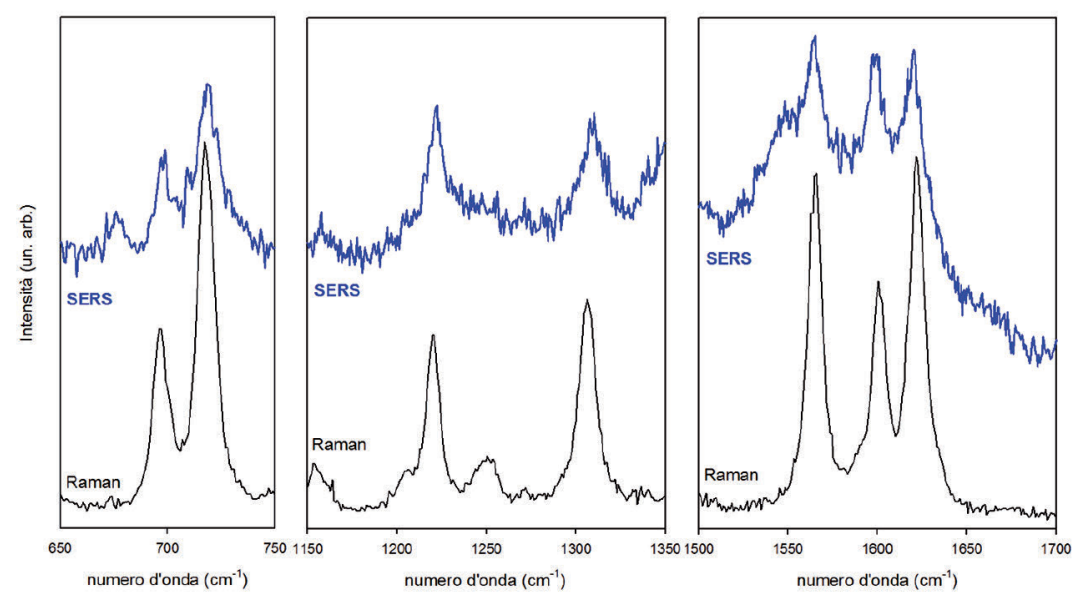

Fig. 10 - Confronto tra lo spettro Raman della carbamazepina in metanolo $(20 \mathrm{mg} / \mathrm{ml}) \mathrm{e}$ lo spettro SERS della carbamazepina $\left(4.2 \times 10^{-4} \mathrm{M}\right)$ acquisito su substrato di oro. Tempo di contatto pari a 60 minuti, volume di soluzione pari a $1 \mathrm{ml}^{15}$

In Fig. 11 sono illustrate in due diverse regioni spettrali, due dei picchi SERS utilizzati come marker di CBZ. L'intensità della risposta SERS in entrambe le regioni dipende dalla concentrazione di CBZ in $\mathrm{MeOH}$. Acquisendo un numero sufficiente di spettri per ciascuna delle concentrazioni studiate è possibile stimare un valore medio di intensità per ciascuno dei segnali SERS selezionati $\left(1621 \mathrm{~cm}^{-1}\right.$ e $\left.717 \mathrm{~cm}^{-1}\right)$. L'andamento di tali valori medi in funzione della concentrazione di CBZ è mostrato in Fig. $12 .{ }^{27}$

In Fig. 13 è mostrato lo spettro SERS registrato da siero di paziente trattato con CBZ, dove sono evidenziati i segnali riconducibili alla presenza del farmaco. ${ }^{27}$

27 A. Bombelli, Dosaggio SERS di farmaci con film nanostrutturati di oro, Tesi di Laurea Magistrale, Politecnico di Milano, 2016. 

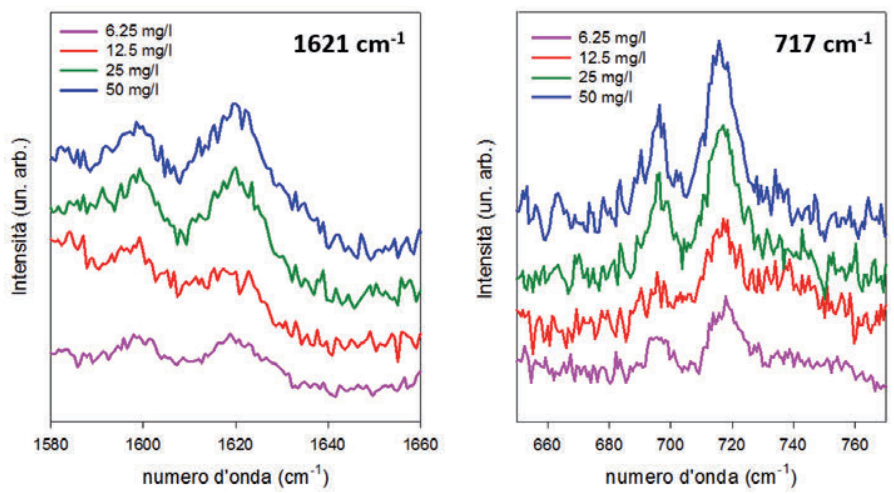

Fig. 11 - Segnali SERS di carbamazepina in due diverse regioni spettrali, in funzione della concentrazione di carbamazepina in $\mathrm{MeOH}(6.25-50 \mathrm{mg} / \mathrm{l})$.
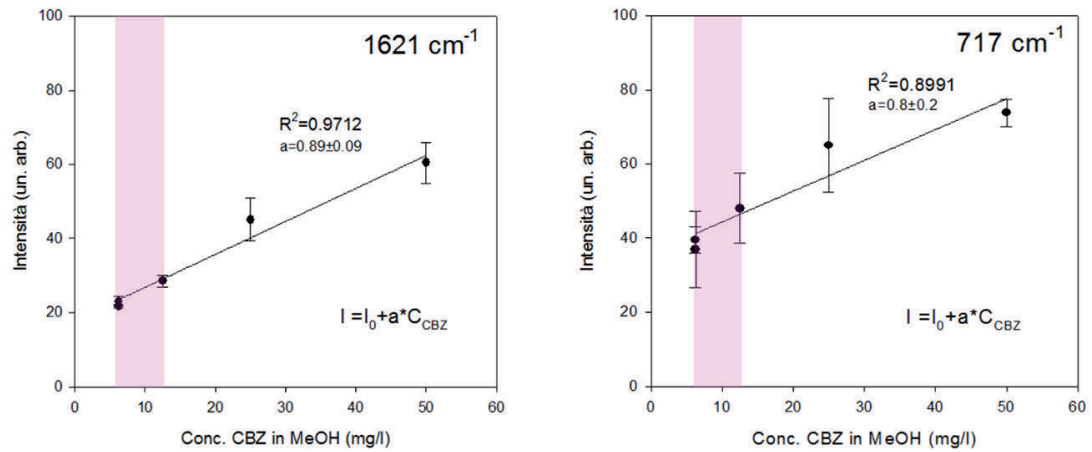

Fig. 12 - Valore medio dell' intensità dei segnali SERS di carbamazepina a $1621 \mathrm{~cm}^{-1}$ e 717 $\mathrm{cm}^{-1}$, in funzione della concentrazione, ottenuti su singolo sensore, lavabile.

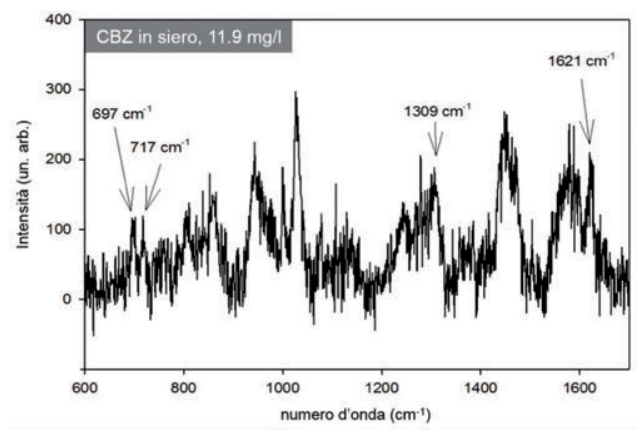

Fig. 13 - Spettro SERS di siero di paziente trattato con carbamazepina. Sono distinguibili segnali riconducibili alla presenza di farmaco $\left(697,717,1309\right.$ e $\left.1621 \mathrm{~cm}^{-1}\right)$. 


\section{CONCLUSIONI}

Abbiamo mostrato come sia possibile produrre in modo controllato, tramite tecnica PLD, film sottili di metalli nobili con proprietà plasmoniche ad hoc per applicazioni in spettroscopia SERS. Al variare di due parametri principali, la pressione di gas ambiente nella camera di deposizione PLD ed il numero di impulsi laser, si possono ottenere superfici nanostrutturate con caratteristiche variabili con continuità da nanoparticelle isolate, ad isole di nanoparticelle progressivamente coalescenti, fino a strutture percolate e film compatti. Nel caso di strutture consistenti in isole di forma irregolare con dimensioni entro poche decine di nanometri, separate fra loro da reti di canali di larghezza nanometrica, le caratteristiche SERS dei film ottenuti sono promettenti, e consentono la rivelazione quantitativa di farmaci di interesse medico clinico, quali Apomorfina e Carbamazepina. In particolare, la misura SERS di Carbamazepina in campioni di plasma sanguigno mostra la misurabilità di concentrazioni di interesse clinico. Il risultato è un buon punto di partenza per correlare l'intensità del segnale SERS del farmaco alla sua concentrazione nel sangue di pazienti, verso la realizzazione di una procedura SERS-TDM complementare alle tecniche esistenti per la quantificazione di farmaci nel sangue.

\section{RINGRAZIAMENTI}

Gli autori sono grati ad Alessandro Bombelli e Claudia Pedrini per i loro contributi alla ricerca ed alla Dott. Francesca Del Sorbo (FERB-Fondazione Europea Ricerca Biomedica, Centro di Riabilitazione Specialistica Neuromotoria, Cernusco s/N (MI)) per le discussioni scientifiche sulla malattia di Parkinson. Questo lavoro è stato parzialmente finanziato dal progetto "Controllare l'Epilessia nei Paesi in via di sviluppo" (Polisocial Award 2014 - Politecnico di Milano). 